\title{
The Functional Outcome of Total Knee Replacement in Young Patients: A 10-Year Matched Case Control Study
}

\author{
Alexander M. Wood, Andre C. M. Keenan, Calum H. C. Arthur, Stuart A. Aitken, \\ Phil J. Walmsley, Ivan J. Brenkel \\ Orthopaedic Department, Victoria Hospital, Kirkcaldy, UK. \\ Email: drsandywood@googlemail.com
}

Received March $20^{\text {th }}, 2013$; revised May $1^{\text {st }}, 2013$; accepted May $19^{\text {th }}, 2013$

Copyright (C) 2013 Alexander M. Wood et al. This is an open access article distributed under the Creative Commons Attribution License, which permits unrestricted use, distribution, and reproduction in any medium, provided the original work is properly cited.

\begin{abstract}
We present the results of a prospective study on the functional outcome from total knee replacement (TKR) in young versus old patients. American Knee Society and pain scores over ten years post TKR were compared using a cohort of young patients ( $\leq 55$ years) and a control group of patients $\geq 56$, matched for ASA, body mass index and preoperative condition. A total of 24 young and 24 older knees were analysed. All scores improved significantly over time. There was no statistical differences over 10 years in pain $(p=0.436)$ and knee performance $(0.618)$. Overall function was consistently higher in the younger group $(p=0.004)$. TKR in younger patients produces similar outcomes in terms of pain and function compared with older patients and we feel that TKR should not be withheld on account of age alone.
\end{abstract}

Keywords: Component; Formatting; Style; Styling; Insert

\section{Introduction}

Total Knee Arthroplasty (TKA) is a proven treatment for severe joint pain in osteoarthritis of the knee. This intervention is well established in the elderly with predictable and reproducible results [1-4]. However, many orthopaedic surgeons are reluctant to perform TKA in younger patients due to the concern that high levels of activity may lead to increasing wear and aseptic loosening. Technical challenges and complications associated with multiple revisions are additional causes for concern in these patients [5]. There are a few reports showing favourable outcomes in the under 55 age group [6-8]. These series contain mainly patients suffering from rheumatoid arthritis and performed by surgeons in specialist centers [9]. It is not clear that these results would be reproducible in general orthopaedic practice [10].

There appears to be no studies comparing the progress of young patients following TKR for osteoarthritis of the knee compared to a control group of matched older patients. The aim of this study was to compare the outcome from total knee replacement (TKR) in young versus old patients in terms of pain and function.

\section{Materials and Methods}

All patients undergoing TKR in our institution since
1995, have been entered prospectively into a database and followed up at regular intervals. From this database we selected patients undergoing TKR who were under the age of 55 years at the primary procedure, regardless of diagnosis. We excluded those, which had not reached at least 10 years follow-up. Once these had been selected, a second control group was selected from the same database of patients, selecting patients who were over the age of $55 \mathrm{yrs}$ at the time of primary procedure, again regardless of diagnosis. The groups were matched for ASA, body mass index and underlying diagnosis.

All patients in the series had demographic and clinical outcome data collected prospectively on admission and at follow up clinics run by a specialist arthroplasty nurse at 10 days pre-operatively and 6 months, 18 months, 3 years, 5 years and 8-10 years post surgery.

The operations were performed by six different consultant surgeons or by a trainee under direct supervision. The operations were performed in a filtered air operating theatre with laminar flow. Waterproof single use gowns and drapes were used and surgeon and assistant were double gloved. A tourniquet was used routinely.

Intramedullary referencing was used for the femoral cuts and extra-medullary referencing for the tibia. All patients in the study received either a PFC (1995-1997) or PFC Sigma CR (1998 onwards) total knee replace- 
ment (Depuy Orthopaedics, Warsaw, IN) through a medial para-patellar approach with the patient supine The patella was not routinely resurfaced, this was only performed when patella wear was severe and the criterion for resurfacing was at the discretion of the operating surgeon. All patients had antibiotic prophylaxis with $1 \mathrm{~g}$ Ceftriaxone shortly before inflation of the tourniquet, pre-and postoperative thromboembolic prophylaxis using low molecular weight heparin and full length graded elastic stockings.

Drains were not used routinely. All surgeons used the same instrumentation and patients underwent the same postoperative regimen. Wounds were dressed with gauze, wool and crepe dressings. These dressings were removed on the first post-operative day and continuous passive mobilisation was commenced. Blood transfusion was only performed if the haemoglobin fell below $8.5 \mathrm{~g} / \mathrm{dl}$. The pre- and postoperative haemoglobin, haemoglobin drop, transfusion received, number of units used, tourniquet time, length of procedure and length of hospital stay (LOS) were noted for each patient.

A transfusion policy was in place throughout the study in which patients with a post-operative haemoglobin level of less than $8.5 \mathrm{~g} / \mathrm{dl}$ were transfused. Patients with a postoperative haemoglobin level of between $8.5 \mathrm{~g} / \mathrm{dl}$ and $10 \mathrm{~g} / \mathrm{dl}$ were only transfused if they displayed clinical symptoms, and those with a haemoglobin level above 10 $\mathrm{g} / \mathrm{dl}$ were not transfused. Pre-operative cross matching of blood was performed only in patients with a pre-operative haemoglobin level below $11 \mathrm{~g} / \mathrm{dl}$.

Change over time was analysed using a factorial repeated measures ANOVA test, which allowed for investigation of difference between groups.

\section{Results}

We identified 40 Knees in 26 patients who were 55 years or younger at the time of primary TKR and within a time frame that would allow 10 years of follow up. Of these 2 patients died prior to final follow up, 2 were revised within the study period. ( 1 for infection within 2 years and one for change of poly at 7 years) and a further 5 were lost to follow up.

35 knees had data at 10 years. These were matched with older patients from the database with 10 year follow up. The groups were matched for BMI, ASA and diagnosis. 7 knees could not be matched and were excluded. This left a study group of 24 young and 24 older knees. 17 of each group suffer from Osteoarthritis and 7 from inflammatory arthritis. This left a study group of 48 patients, 24 young and 24 older knees.

The average age for the younger group was 49.9 with a range of (38 - 55) and the older 69.7 (range 57 - 84). The average BMI for each group was 29 (Table 1).
Table 1. It shows patient demographics.

\begin{tabular}{lcc}
\hline & Young & Older \\
\hline Average Age & 49.9 & 69.7 \\
Median age & 53 & 71 \\
Range & $38-55$ & $57-84$ \\
Average BMI & 29.3 & 29.7 \\
Male & 16 & 10 \\
Female & 8 & 14 \\
Bilateral & 9 & 6 \\
Osteoarthritis & 17 & 17 \\
Inflammatory & 7 & 7 \\
\hline
\end{tabular}

Average Length of stay was higher for the older group 10.9 days compared to the younger group 8.13 days. This trend continued in both unilateral and bilateral patient groups, with bilateral replacements patients stayed an average of 9.0 days in the young group compared with 11.3 in the older group in contrast to 7.7 and 10.8 days in the unilateral knee replacement group.

Pain scores $(p=0.025)$ (Figure 1) and American Knee Society "Knee" ( $p<0.001)$ (Figure 2$)$ and "Function" ( $p<$ 0.001 ) scores (Figure 3 ) changed significantly over time. There were however no statistical differences over the 10 year period in pain $(\mathrm{p}=0.436)$ and knee performance (0.618) but overall function was higher throughout the period in the younger group $(=0.004)$.

The greatest difference is seen in the function scores with the young group scoring 69.3 at ten years and the older group scoring $54.5(\mathrm{p}<0.05)$. It would appear that the younger patients achieve and maintain a higher function after TKA. A decline is seen between 5 and ten years that is reflected in the older group also. Both groups are seen to decline at 10 years post arthroplasty but the reasons for this are not clear. There is no increase in the revision rate for either group at this time. The decline may reflect a change in the activity profile of patients in both groups who are, by definition, 10 years older.

The function scores are better for the younger knees and remain so for longer. This difference may be due to the general decline in mobility with advancing years. For example the patient who uses a stick will lose points.

\section{Discussion}

The aim of this study was to compare functional outcomes for young patients undergoing TKA with older patients. The population at risk is likely to remain fit and active for longer and many are keen to maintain an active lifestyle despite joint disease [11]. As a result younger patients are presenting to orthopaedics services requesting joint replacement [12]. 


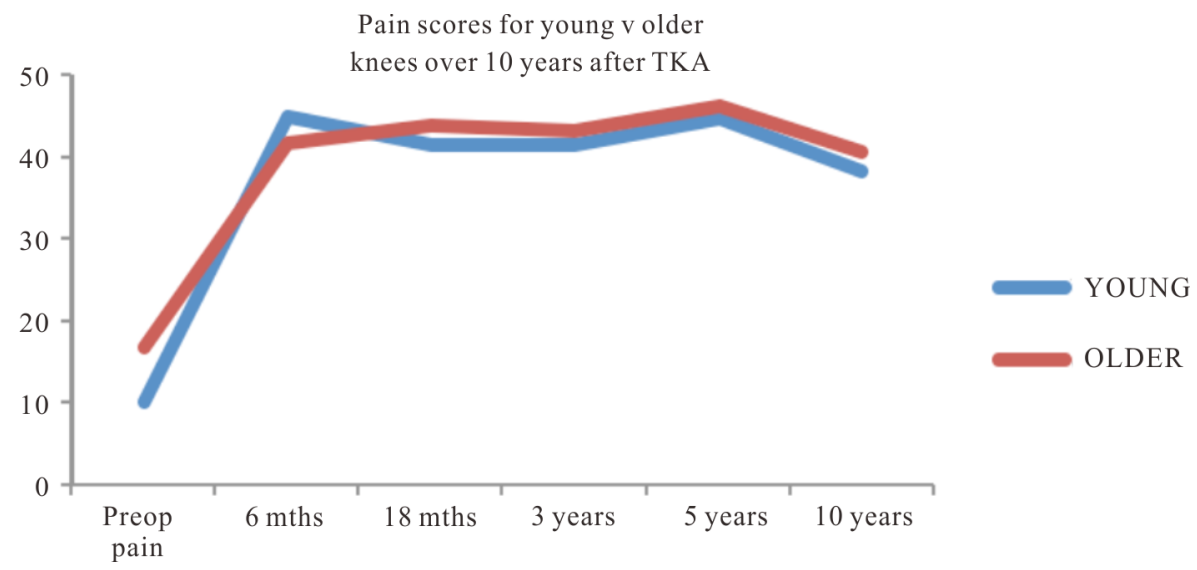

Figure 1. Pain scores.

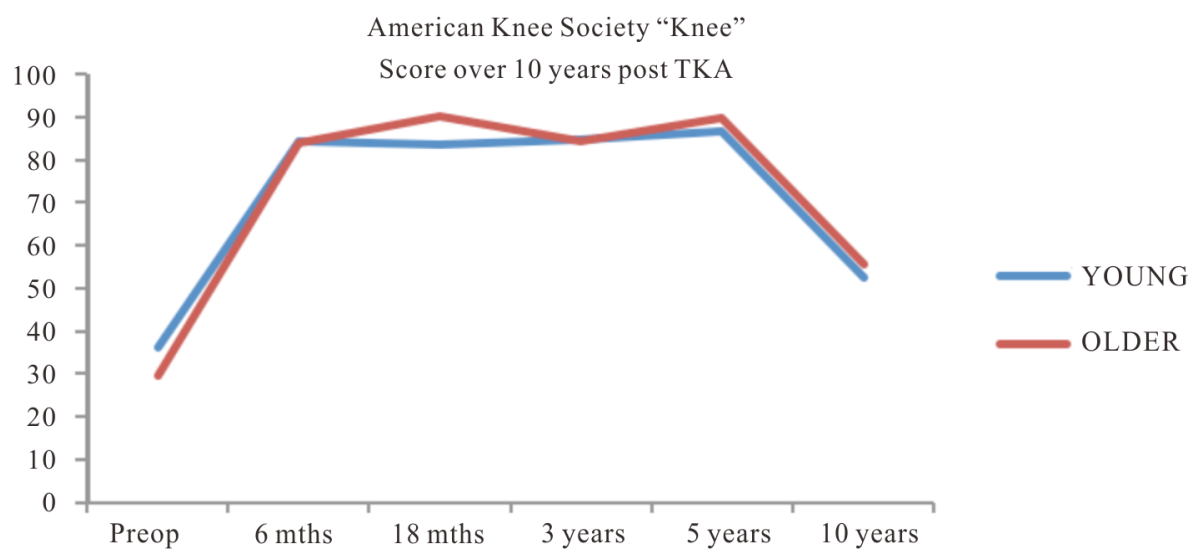

Figure 2. American knee society scores.

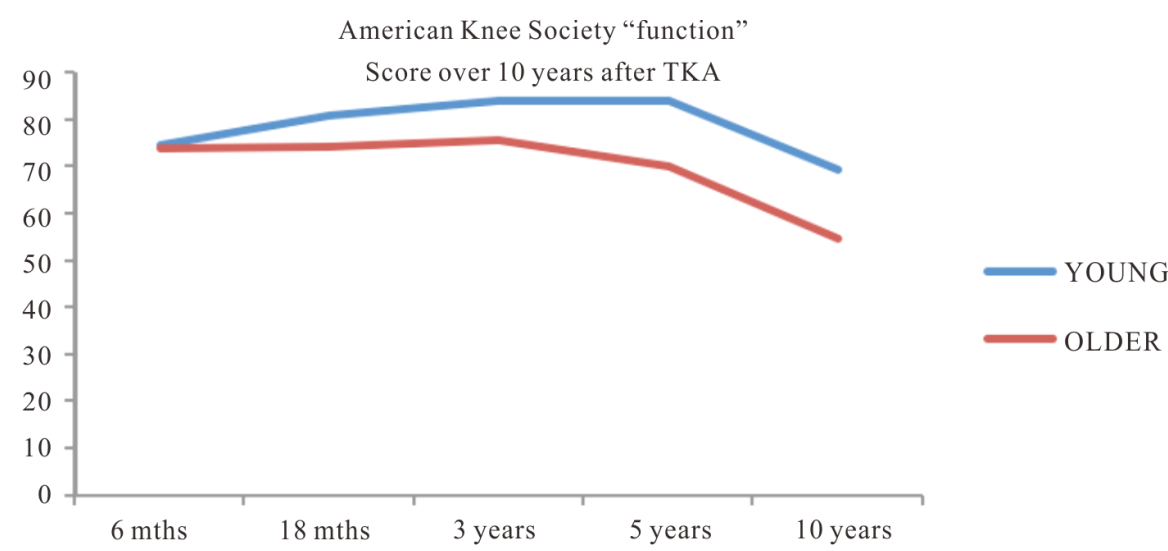

Figure 3. Function scores.

TKA is routinely performed in older patients with osteoarthritis of the knee. There are reservations about offering this procedure to younger patients as their higher demands may lead to premature failure and subsequent revision. Harrysson et al. looked at large cohort of patients and demonstrated higher revision risk for patient under 60 compared to their older counterpart. They note that the risk is related to the year of surgery and better results may be seen with newer implant technology [5] Gloe et al. demonstrated a 14 year survival of $85 \%$ of cemented TKA in those under 55year old. They showed revision risk was unaffected by younger age or diagnosis. [10]

A systematic review reported Implant survivorship between $90.6 \%$ and $99 \%$ during the first decade and between $85 \%$ and $96.5 \%$ during the second decade of fol- 
low-up. Mean American Knee Society clinical and functional scores increased by 47 and 37 points [7]. Ranawat et al report on a cohort of younger patients, Most of who suffered from rheumatoid arthritis. They highlight a radiographic lucency in $30 \%$ but it is unclear the clinical significance of this as they report a ten year survival of $96 \%$.

The data presented here suggests that recipients of TKA gain similar functional \& subjective benefit regardless of age and follow a similar course in the years following surgery. It is suggested from the results of our study that the younger patients achieve and maintain a higher function after TKA. The function scores are better for the younger knees and remain so for longer. Overall function was higher throughout the period in the younger group $(\mathrm{p}=0.004)$. This difference may be due to the general decline in mobility with advancing years rather than specifically relating to TKA. It is important to note that both groups are seen to decline at 10 years post arthroplasty. This may herald an increase in the rate of revision for both groups as at $10-15 \mathrm{yr}$. There is not a marked decline in AKSS in the younger patients compared to the control. The trends between both groups remain similar with a decline between 5 and 10 years. We have make no observation regarding survivorship in either group as this lies out-with the scope of this study which was to look at the matched trends pain and functional outcome following TKA in patients under 55years of age. We aim to provide a separate study looking at survivorship of TKA at 10 years post procedure.

Our results suggest younger patients achieve similar results from TKA, in terms of pain and function, which are comparable and even superior to older patients when matched for ASA, Diagnosis and BMI. This agrees with other evidence suggesting good or excellent results after TKA $[7,10,13]$. Care must be taken in interpreting these results as a small number of patients were available at 10 years for review and represents a potential weakness in our study. This potential source of error may have occurred by those with poor outcomes self-selecting out by not returning for follow up.

The data does show a decline in AKSS between five and ten years. This may represent the beginning of potential problems for the younger patients with TKA. However it is interesting to note that this is not peculiar to the younger patients and this trend is seen in the matched controls as well. It is clear that this cohort of younger patients gained significant benefit from TKA continued to have good function at ten years of follow up. This highlights the importance of patient selection preoperatively and setting realistic expectations of patients we consent for surgery. We plan to continue to observe this cohort through their second decade post TKA to see how the pain and function alters and when they come to revision.
Patients aged less than 55 years undergoing TKA can achieve similar outcomes at 10 years post procedure in terms of pain and function compared with older patients when matched for ASA grade, BMI \& diagnosis. This information is useful in counseling and consenting patients prior to surgery. Careful patient selection remains critical in achieving these outcomes. We suggest that TKA should not be withheld in patients under 55 years of age purely on grounds of age alone.

\section{Acknowledgements}

We would like to thank Anne Simpson, Lorraine Mc Cominskie, Wilma Addison and Janette MacDonald and for their hard work in the data collection in the follow up clinics.

Conflict of interest statement: None of the authors has received or will receive benefits for personal or professional use from a commercial party directly or indirectly related to the subject of this article. However, benefits have been received which have been solely directed to a non-profit making research fund with which one or more of the authors are associated.

\section{REFERENCES}

[1] L. K. George, D. J. Ruiz and F. A. Sloan, "The Effects of Total Knee Arthroplasty on Physical Functioning in the Older Population," Arthritis \& Rheumatism, Vol. 58, No. 10, 2008, pp. 3166-3171. doi:10.1002/art.23888

[2] C. M. Callahan, B. G. Drake, D. A. Heck and R. S. Dittus, "Patient Outcomes Following Tricompartmental Total Knee Replacement: A Meta-Analysis," JAMA, Vol. 271, No. 17, 1994, pp. 1349-1357. doi:10.1001/jama.1994.03510410061034

[3] F. H. Norman-Taylor, C. R. Palmer and R. N. Villar, "Quality-of-Life Improvement Compared After Hip and Knee Replacement," The Bone \& Joint Journal, Vol. 78, No. 1, 1996, pp. 74-77.

[4] C. H. Arthur, A. M. Wood, A. C. Keenan, R. A. Clayton, P. Walmsley and I. Brenkel, "Ten-Year Results of the Press Fit Condylar Sigma Total Knee Replacement," The Bone \& Joint Journal, Vol. 95, No. B-2, 2013, pp. 177180. doi:10.1302/0301-620X.95B2.29695

[5] O. Harrysson, O. Robertsson and J. Nayfeh, "Higher Cumulative Revision Rate of Knee Arthroplasties in Younger Patients with Osteoarthritis," Clinical Orthopaedics and Related Research, No. 421, 2004, pp. $162-$ 168. doi:10.1097/01.blo.0000127115.05754.ce

[6] C. S. Ranawat, D. Padgett and Y. Ohashi, "Total Knee Arthroplasty for Patients Younger than 55 Years," Clinical Orthopaedics and Related Research, No. 248, 1989, pp. 27-33.

[7] J. Keeney, S. Eunice, G. Pashos, R. Wright and J. Clohisy, "What Is the Evidence for Total Knee Arthroplasty in Young Patients? A Systematic Review of the Literature," 
Clinical Orthopaedics and Related Research, Vol. 469, No. 2, 2011, pp. 574-583.

doi:10.1007/s11999-010-1536-9

[8] D. R. Diduch, J. N. Insall, W. N. Scott, G. R. Scuderi and D. Font-Rodriquez, "Total Knee Replacement in Young, Active Patients: Long-Term Follow-Up and Functional Outcome," The Journal of Bone \& Joint Surgery, Vol. 79, No. 4, 1997, pp. 575-582.

[9] A. C. M. Keenan, A. M. Wood, C. A. Arthur, P. J. Jenkins, I. J. Brenkel and P. J. Walmsley, "Ten-Year Survival of Cemented Total Knee Replacement in Patients Aged Less than 55 Years," The Journal of Bone \& Joint Surgery, Vol. 94, No. 7, 2012, pp. 928-931. doi:10.1302/0301-620X.94B7.27031

[10] T. Gioe, C. Novak, P. Sinner, W. Ma and S. Mehle, "Knee Arthroplasty in the Young Patient: Survival in a
Community Registry," Clinical Orthopaedics and Related Research, Vol. 464, 2007, pp. 83-87.

[11] C. J. Lavernia, J. C. Alcerro and J. S. Contreras, "Knee Arthroplasty: Growing Trends and Future Problems," International Journal of Clinical Rheumatology, Vol. 5, No. 5, 2010, pp. 565-579. doi:10.2217/ijr.10.49

[12] J. A. Singh, M. B. Vessely, S. W. M. S. Harmsen, et al., "A Population-Based Study of Trends in the Use of Total Hip and Total Knee Arthroplasty, 1969-2008," Mayo Clinic Proceedings, Vol. 85, No. 10, 2010, pp. 898-904. doi: $10.4065 / \mathrm{mcp} .2010 .0115$

[13] C. S. Ranawat, D. E. Padgett and Y. Ohashi, "Total Knee Arthroplasty for Patients Younger than 55 Years," Clinical Orthopaedics and Related Research, No. 248, 1989, pp. 27-33. 